\title{
Cortico-pontine theta carrier frequency phase shift across sleep/wake states following monoaminergic lesion in rat
}

\author{
Aleksandar Kalauzi ${ }^{1}$, Sladjana Spasic ${ }^{1}$, Jelena Petrovic ${ }^{2}$, Jelena Ciric $^{2}$ and Jasna Saponjic ${ }^{2}$ \\ ${ }^{1}$ University of Belgrade, Department of Life Sciences, Institute for Multidisciplinary Research, 11030, Belgrade, Serbia \\ ${ }^{2}$ University of Belgrade, Department of Neurobiology, Institute for Biological Research-Sinisa Stankovic, 11060, Belgrade, \\ Serbia
}

\begin{abstract}
This study was aimed to explore the sleep/wake states related cortico-pontine theta carrier frequency phase shift following a systemically induced chemical axotomy of the monoaminergic afferents within a brain of the freely moving rats. Our experiments were performed in 14 adult, male Sprague Dawley rats, chronically implanted for sleep recording. We recorded sleep during baseline condition, following sham injection (saline i.p. $1 \mathrm{ml} / \mathrm{kg}$ ), and every week for 5 weeks following injection of the systemic neurotoxins (DSP-4 or PCA; $1 \mathrm{ml} / \mathrm{kg}$, i.p.) for chemical axotomy of the locus coeruleus (LC) and dorsal raphe (DR) axon terminals. After sleep/wake states identification, FFT analysis was performed on $5 \mathrm{~s}$ epochs. Theta carrier frequency phase shift $(\Delta \Phi)$ was calculated for each epoch by averaging theta Fourier component phase shifts, and the $\Delta \Phi$ values were plotted for each rat in control condition and 28 days following the monoaminergic lesions, as a time for permanently established DR or LC chemical axotomy. Calculated group averages have shown that $\Delta \Phi$ increased between pons and cortex significantly in all sleep/wake states (Wake, NREM and REM) following the monoaminergic lesions, with respect to controls. Monoaminergic lesions established the pontine leading role in the brain theta oscillations during all sleep/wake states.
\end{abstract}

Key words: Monoaminergic lesion - Sleep/wake states - Theta carrier frequency phase shift - Locus coeruleus - Nucleus dorsalis raphe

\section{Introduction}

Neuronal network oscillations in the brain functions, and neuronal synchrony within the same network or between interrelated networks, have been extensively investigated on different oscillation bands (Singer 1993). However, the exact mechanisms of brain oscillations and synchronization of neuronal activity within single or between different brain structures are not well understood (Penttonen and Buzsaki 2003).

The hippocampal theta rhythm is the most studied rhythm in rat. Theta oscillations occur across many brain

Correspondence to: Jasna Saponjic, University of Belgrade, Department of Neurobiology, Institute for Biological Research-Sinisa Stankovic, Despot Stefan Blvd. 142, 11060 Belgrade, Serbia

E-mail: jasnasap@ibiss.bg.ac.rs jasnasaponjic@yahoo.com structures, like within the structures of the limbic cortex (Buzsaki 2002). They are present in the hippocampal and retrohippocampal areas in the cat, rabbit, and human (Vertes and Kocsis 1997), and they are considered as a correlate of arousal, orientation, exploration, attention, learning and memory, motivational drives, emotions, voluntary movements, the encoding of new information (Vinogradova 1995).

Ascending brainstem synchronizing pathways influence the electrophysiological and pharmacological properties of the neocortex, and their functional significance is generalized regulation of neocortical activities related to sensorimotor behavior (Vertes 1982; Bland and Oddie 1998).

It is well known that the pontine cholinergic neurons of the pedunculopontine tegmental nucleus/laterodorsal tegmental nucleus (PPT/LDT), as a part of the ascending brainstem synchronizing pathway (Karashima et al. 2004; Datta and McLean 2007), play a crucial role in generation 
of rapid eye movement (REM) sleep through reciprocal interconnection with serotonergic dorsal raphe (DR) and noradrenergic locus coeruleus (LC) neurons (Hobson et al. 1975). Inhibitory interaction between these regions are important in regulating their activity, and the opposite, reciprocal discharge patterns of the monoaminergic and cholinergic neurons within pons were proposed to underline the cyclic appearance of REM sleep (McCarley and Hobson 1975). DR and LC are the main sources of monoamines in the brain, and their extensive efferents implicate these structures in a variety of behavioral functions, particularly in sleep regulation (Lydic et al. 1987), motor (Fenik and Veasey 2003) and respiratory control (Carley and Radulovacki 2003; Saponjic et al. 2005).

It was demonstrated that the systemic selective lesion of the noradrenergic or serotonergic axon terminals did not affect the sleep/wake distribution compared with controls (Saponjic et al. 2007a). Using spectral analysis and phase shift spectra of the cortical and pontine electroencephalogram (EEG), we have proved that chronically decreased brain monoamines in freely moving rats changed synchronization phase shift between sensorimotor cortical and pontine theta oscillations, and pons became the leading theta oscillator (Saponjic et al. 2007b; Kalauzi et al. 2008, 2009). Although a tonically decreased level of brain monoamines did not induce any disturbance in sleep/wake distribution, it induced a characteristic mode of sigma/theta coupling during non REM (NREM) sleep, suggesting an importance of monoamines in NREM sleep consolidation (Kesic et al. 2011). All these results suggest an importance of monoamines in control of sleep spindle and theta rhythm generators, as well as in the brain theta oscillation synchronization.

This study was aimed to explore the sleep/wake states related cortico-pontine theta carrier frequency phase shift following systemically induced chemical axotomy of monoaminergic afferents within the brain of freely moving rats.

\section{Materials and Methods}

The experiments were performed in 14 adult, male Sprague Dawley rats chronically implanted for sleep recording, during baseline condition, following sham injection (saline i.p. $1 \mathrm{ml} / \mathrm{kg}$ ), and every week for 5 weeks following injection of the systemic neurotoxins. The surgical procedures employed for chronic electrode implantation, and all details about systemically induced monoaminergic axotomy (the monoaminergic lesions) have been previously described (Saponjic et al. 2007a), and are outlined below.

Prior to surgery and consistently throughout the experimental protocol, animals were maintained on a 12- hour light-dark cycle, and were housed at $25^{\circ} \mathrm{C}$ with free access to food and water. Principles for the care and use of laboratory animals in research were strictly followed, as outlined by the Guide for Care and Use of Laboratory animals (National Academy of Sciences Press, Washington DC, 1996).

For the operative procedure animals were anesthetized with a combination of $80 \mathrm{mg} / \mathrm{kg}$ ketamine (Abbot Laboratories, North Chicago, IL, USA) and $5 \mathrm{mg} / \mathrm{kg}$ xylazine (Phoenix Scientific Inc. St. Joseph, MO, USA) by intraperitoneal (i.p.) injection. We implanted bilateral parietal stainless-steel screw electrodes for EEG cortical activity recording (A/P: 2.5; R/L: 2; D/V: 1 to bregma), and a twistedpair bipolar electrode, fabricated using teflon coated wire (Medwire, Mt. Vernon, NY, USA) with an uninsulated tip of $0.5 \mathrm{~mm}$, was stereotaxically targeted into the right PPT (A/P: $-7.80 ; \mathrm{L}: 2 ; \mathrm{D} / \mathrm{V}:-7.40$ ) for pontine P-wave recording, and bilateral electromyogram (EMG) wire electrodes were implanted into the dorsal nuchal musculature to assess skeletal muscle activity. All electrode leads were soldered to a miniature connector plug (39F1401, Newark Electronics, Schaumburg, Illinois, USA), and the assembly was fixed to the screw electrodes and skull using acrylic dental cement (Justi, Saslow Dental, Mt. Prospect, Illinois, USA). Scalp wounds were sutured and the rats were recovered for 2 weeks before experimental recording sessions began. All experimental recordings were bipolar and began at 9:00 a.m. and lasted $6 \mathrm{~h}$. EEG activities carried from the connector plug on the rat head by cable and passed through a sealed port of the plethysmograph were displayed on a computer monitor (Experimenter's Workbench, Datawave Systems, Longmont, CO, USA), and stored on disk for further offline analysis. After conventional amplification and filtering (0.1-50 Hz band pass; Grass Model 12, West Warwick, RI) the analog data were digitized (sampling frequency 100/s) and recorded using Brain Wave for Windows software (Datawave System Longmont, CO, USA).

For chemical axotomy of the locus coeruleus $(n=7)$ we used DSP-4 (N-(2-chloroethyl)-N-ethyl-2-bromomenzilamine, Sigma-Aldrich, MO, $50 \mathrm{mg} / \mathrm{kg}$ i.p. in single dose), and for dorsal raphe axon terminals $(n=7)$ PCA neurotoxin (p-chloroamphetamine, Sigma-Aldrich, MO, two i.p. injections, $6 \mathrm{mg} / \mathrm{kg}$, $24 \mathrm{~h}$ apart).

In this study, for analysis of the lesion effects, we included only recordings from animals that completed the 5 -week post-lesion follow-up period (4 PCA lesioned rats, and 5 DSP-4 lesioned rats).

For the spectral and phase shift analysis we used overall 6-h recordings in control condition and 28 days following monoaminergic lesions, as a well evidenced time for permanently established DR or LC chemical axotomy. Each lesioned rat was compared with respect to its own control. Sleep/wake states (Wake, NREM, REM) were identified for 
A
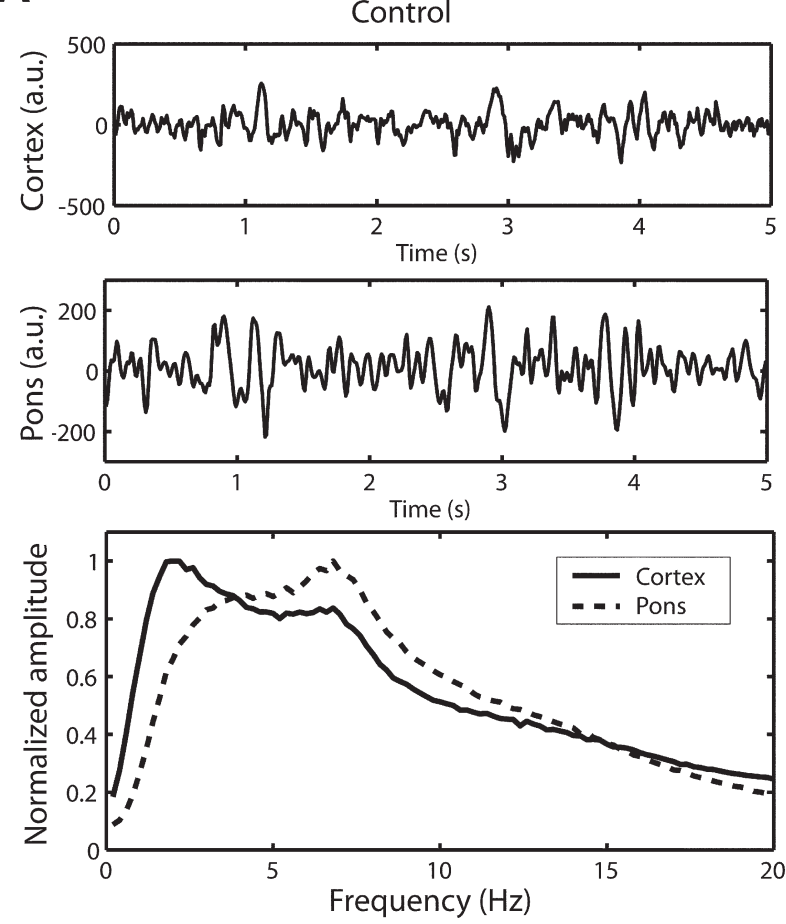

B
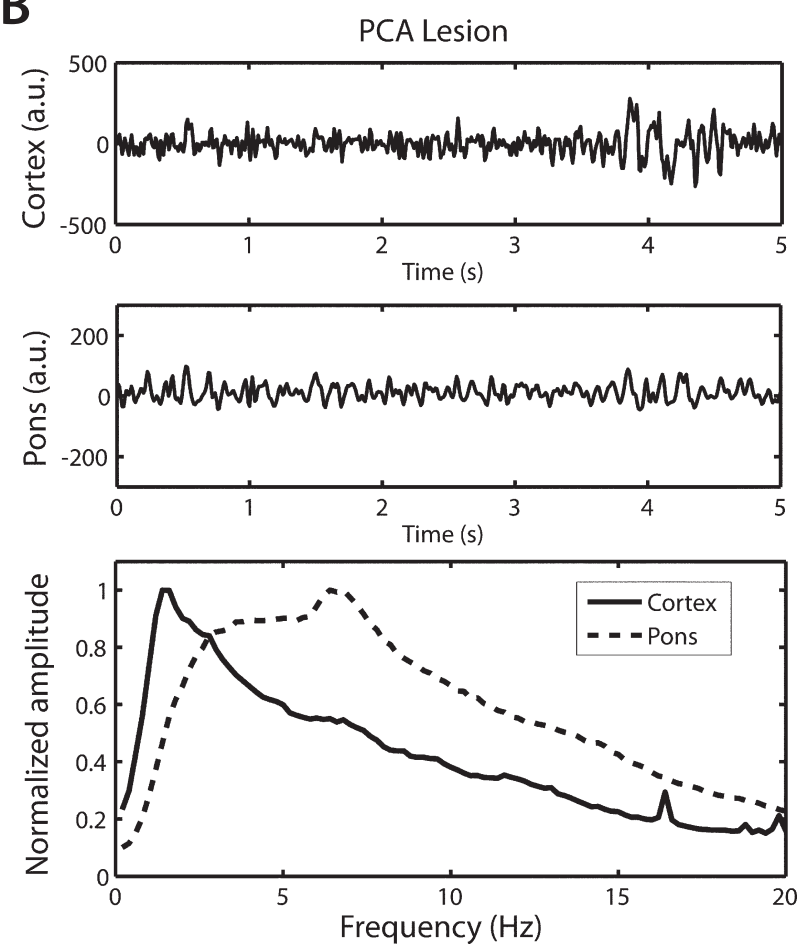

Figure 1. Analog signals and amplitude spectra from an individual rat in control condition (A) and 28 days following PCA lesion (B). Upper panels: an example of $5 \mathrm{~s}$ epoch of analog cortical (Cortex) and pontine (Pons) EEG signal. Lower panels: mean normalized amplitude spectra of the $6 \mathrm{~h}$ recordings ( 4320 epochs of $5 \mathrm{~s}$ per each signal and condition) depict the characteristic overlapping of cortical and pontine spectra in the amplitude of 5-8 Hz band. PCA, p-chloramphetamine.

each $10 \mathrm{~s}$ epoch. We extracted all $5 \mathrm{~s}$ epochs (4320 epochs of $5 \mathrm{~s}$ ) from cortical and pontine EEG signals (upper panels Fig. 1A,B), and the Fourier analysis was performed using standard FFT routine supplied with MATLAB 6.5. Amplitude spectra were calculated for each $5 \mathrm{~s}$ epoch (lower panels Fig. 1A,B) and averaged. In order to calculate the theta frequency phase shifts, we used our originally developed procedure, described in all details in Kalauzi et al. (2009), where we treated each of the two signals in the theta band as amplitude modulated carrier frequency signals.

Namely, we observed each of the two signals in the theta band as amplitude modulated carrier frequency sinusoidal functions:

$$
u(t)=\left(1+u_{m}(t)\right) A_{c} \sin \left(\omega_{c} t+\Phi\right)
$$

where $u_{m}$ represents the modulating waveform; $A_{c}, \omega_{c}$ and $\Phi$ stand for the amplitude, circular frequency and initial phase of the carrier wave, respectively. By expanding the modulating waveform into Fourier series

$$
u_{m}(t)=\sum_{i=1}^{n} U_{m i} \sin \left(\omega_{m i} t+\varphi_{m i}\right)
$$

Eq. (1) could be rewritten as:

$$
u(t)=A_{c} \sin \left(\omega_{c} t+\Phi\right)+A_{c} \sin \left(\omega_{c} t+\Phi\right) \sum_{i=1}^{n} U_{m i} \sin \left(\omega_{m i} t+\varphi_{m i}\right)
$$

By transforming each product of sinuses in Eq. (3) into a sum, we obtain

$$
\begin{aligned}
& u(t)=A_{c} \sin \left(\omega_{c} t+\Phi\right)+\frac{1}{2} \sum_{i=1}^{n} U_{m i}\left\{\operatorname { c o s } \left[\left(\omega_{c}-\omega_{m i}\right)\right.\right. \\
& \left.\left.t+\Phi-\varphi_{m i}\right]-\cos \left[\left(\omega_{c}+\omega_{m i}\right) t+\Phi+\varphi_{m i}\right]\right\}
\end{aligned}
$$

Eq. (4) shows that each EEG oscillation, being characterized by variable amplitude, occupies a frequency band symmetrically around the carrier frequency $\omega_{c}$, usually coinciding with position of the local band maximum in the amplitude spectrum. More, this equation shows that initial phase of the carrier frequency, $\Phi$, could be calculated by averaging initial phases of Fourier components from the lower and higher side bands of the modulated signal, symmetrically around $\omega_{c}$. The same is valid if we record two amplitude variable EEG signals with equal carrier frequencies; their carrier 
frequency phase shift $(\Delta \Phi)$ could be obtained by averaging phase shifts of Fourier components, $\Delta \Phi \pm \Delta \varphi_{m i}$. Bearing in mind that phases and phase differences are angular random variables, amplitude-weighted angular average for calculating $\Delta \Phi$ should be applied:

$$
\Delta \Phi=\arctan \frac{\sum_{i=1}^{n} A_{1 i} A_{2 i} \sin \left(\Delta \varphi_{i}\right)}{\sum_{i=1}^{n} A_{1 i} A_{2 i} \cos \left(\Delta \varphi_{i}\right)}
$$

where $A_{1 \mathrm{i}}$ and $A_{2 \mathrm{i}}$ stand for amplitudes of the $i$-th Fourier component of the two signals (indexed by 1 and 2). As can be seen from formula (5), carrier frequency phase shift, $\Delta \Phi$ is not sensitive to changes of amplitudes of any of the two signals, since signal attenuation or amplification is directly proportional to all Fourier amplitudes. Consequently, during this analysis, there was no need to track the degree of amplification or perform signal amplitude calibration.

In our experiments, theta carrier frequency phase shifts $(\Delta \Phi)$ were obtained, for each of the corresponding number of epochs, by integrating Fourier component phase shifts in the range $4.2-8 \mathrm{~Hz}$, and plotted, according to procedure explained above, and in Kalauzi et al. (2009). Carrier frequencies for all rats were in the range 5.2-6.8 Hz. Mean values of carrier frequency phase shifts, mean $\Delta \Phi$, calculated from each histogram, were then transformed into corresponding time delays for each theta carrier frequency $c f_{\theta}$ by applying formula $t_{D}=$ (mean $\Delta \Phi) /\left(c f_{\theta} \times 360^{\circ}\right)$. In angular statistics, phase locking between two oscillations is significant if standard deviation of their phase difference falls below a critical value. Angular significances for theta carrier $\Delta \Phi$ locking, at probability level $p$, were estimated by comparing values of the detected angular standard deviations (SD $\Delta \Phi$ ) with their equivalents for random angles (for 4320 epochs the significantly critical, at the level $p<0.05$, was SD $\Delta \Phi<44.67 \mathrm{deg}$ ).

\section{Results}

We formerly detected (Kalauzi et al. 2009) significant $\Delta \Phi$ locking between cortical and pontine theta carrier fre-

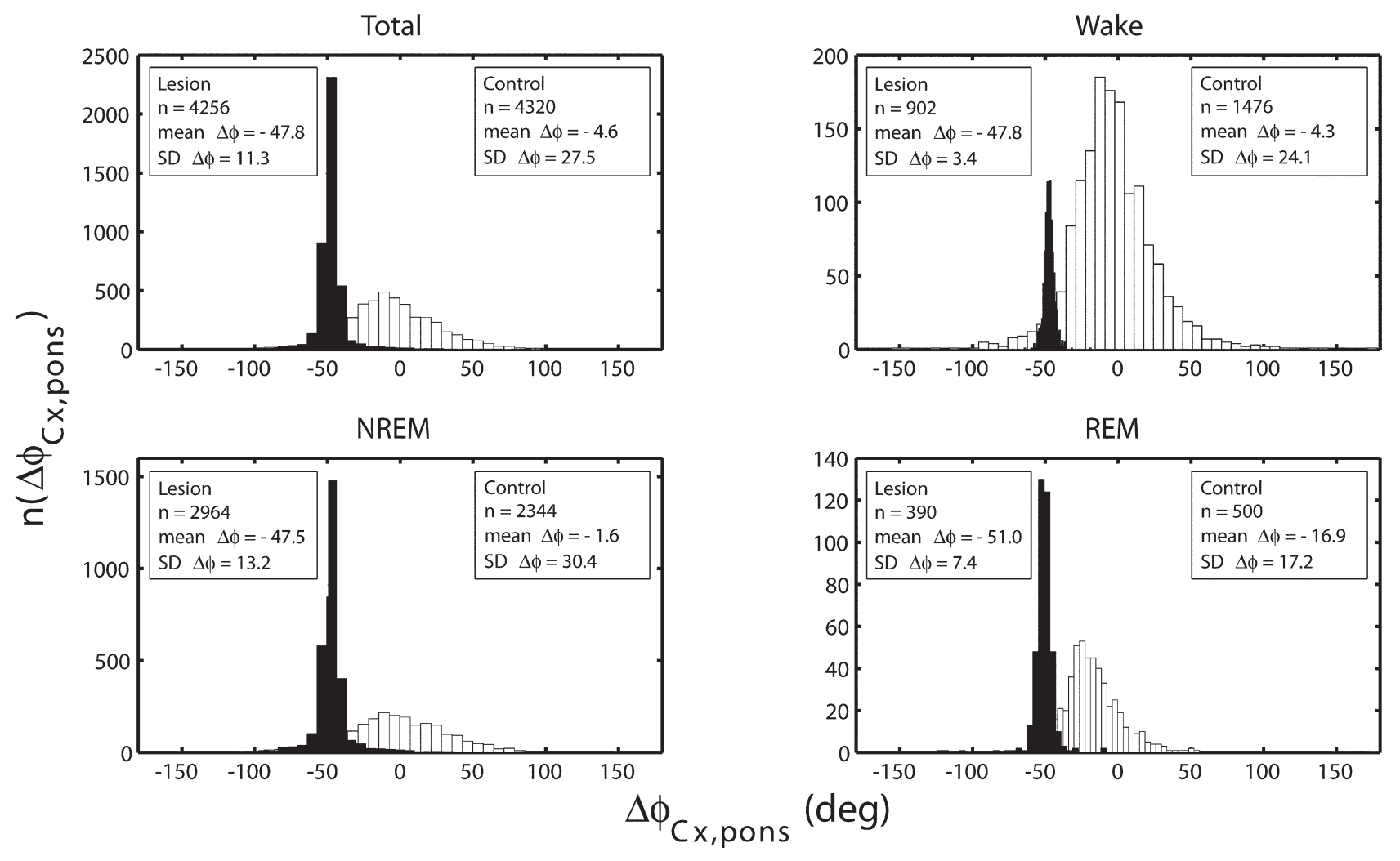

Figure 2. Individual examples of the cortico-pontine theta carrier frequency phase shift histograms per $6 \mathrm{~h}$ of sleep recording (Total), and in each sleep/wake state during 6 h of sleep recording (Wake, NREM, REM). White: control histogram. Black: 28 days after PCA lesion. Number of detected epochs (n) for each state, the angular $\Delta \Phi$ mean, and its corresponding standard deviation are indicated in the upper left/right corner of each. $\Delta \Phi_{\mathrm{Cx} \text {,pons }}$, theta carrier frequency phase shift; $\mathrm{n}\left(\Delta \Phi_{\mathrm{Cx} \text {,pons }}\right)$, number of epochs having $\Delta \Phi_{\mathrm{Cx} \text {,pons }}$ within each histogram bin. 
quency in 6/9 rats, determined for each rat in the range 5.2-6.8 Hz, in both conditions (control and lesion), and an augmentation of the theta carrier phase shift towards pons leading in phase compared to cortex. Here we have followed $\Delta \Phi$ across differentiated sleep/wake states for these six animals. Individual examples of $\Delta \Phi$ histograms per $6 \mathrm{~h}$ of recording, in total time (Total), and extracted per each sleep/wake state (Wake; NREM; REM) in control condition (white histograms), and 28 days following lesion (black histograms) are presented on Fig. 2 (for PCA lesion) and Fig. 3 (for DSP-4 lesion). Sleep/wake states differentiated $\Delta \Phi$ have shown that in control condition in $3 / 6$ rats cortical theta preceded pontine, while in $3 / 6$ rats it was the opposite in all sleep stages (Wake, NREM and REM) (Fig. 4). Following lesion, pontine theta leaded in $4 / 6$ rats during Wake, in 5/6 rats during NREM and REM sleep. When we compared Wake, NREM and REM group mean $\Delta \Phi$ values by Friedman ANOVA test we evidenced a significant lesion induced leading pontine theta with respect to control condition for all sleep states $\left(6 / 6 ; \chi^{2}=\right.$ $21.90, p<0.0006)$. Control group $\Delta \Phi$ means were: for Wake
$13.38 \pm 40.26$ deg; for NREM $19.88 \pm 37.24 \mathrm{deg}$; and for REM $13.83 \pm 41.73 \mathrm{deg}$. Lesion group $\Delta \Phi$ means were: for Wake $-42.22 \pm 57.65 \mathrm{deg}$; for NREM $-42.88 \pm 38.86 \mathrm{deg}$; and for REM $-37.75 \pm 54.24 \mathrm{deg}$. Therefore, positive $\Delta \Phi$ (cortex leading in phase) in control rats changed its sign, signifying that pons became the leading structure in all states and in both lesions. There was no significant difference between state related group $\Delta \Phi$ means in control $(\mathrm{z} \geq-0.41, p=0.68)$, and lesion conditions $(\mathrm{z} \geq-0.41, p \geq$ 0.22 ), but the group $\Delta \Phi$ means of separate Wake, NREM and REM in lesion condition were significantly different with respect their control values $(\mathrm{z}=2.04, p=0.04)$. All individual values of control and lesion mean $\Delta \Phi$ for Wake, NREM and REM are depicted on Table 1.

When we analyzed the group mean $\Delta \Phi$ differences between control and lesion conditions (Fig. 5) for all sleep/wake states, we have found the highest group mean $\Delta \Phi$ difference value in NREM sleep (Wake - 55.6 \pm 20.30 deg; NREM - $62.8 \pm 37.33 \mathrm{deg} ;$ REM - $51.6 \pm 19.22 \mathrm{deg})$, but we did not evidence this difference as statistically significant $\left(\chi^{2}=3.0, p<0.22\right)$. All individual $\Delta \Phi$ differ-
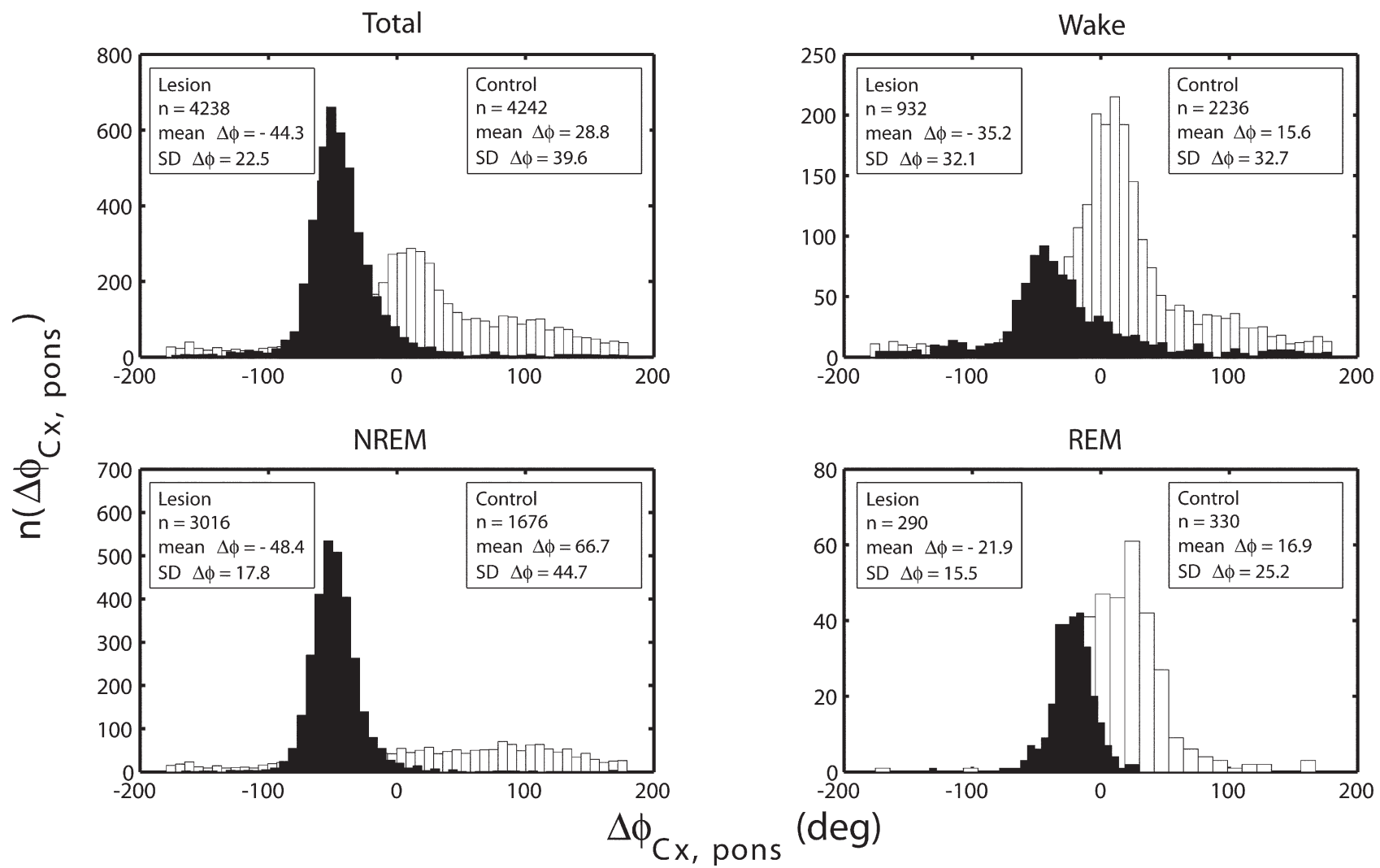

Figure 3. Individual examples of the cortico-pontine theta carrier frequency phase shift histograms per $6 \mathrm{~h}$ of sleep recording (Total) and each sleep/wake state during $6 \mathrm{~h}$ recording (Wake, NREM, REM). White: control histogram. Black: 28 days after DSP-4 lesion. Number of detected epochs (n) for each state, the angular $\Delta \Phi$ mean, and its corresponding standard deviation are indicated in the upper left/right corner of each histogram. 

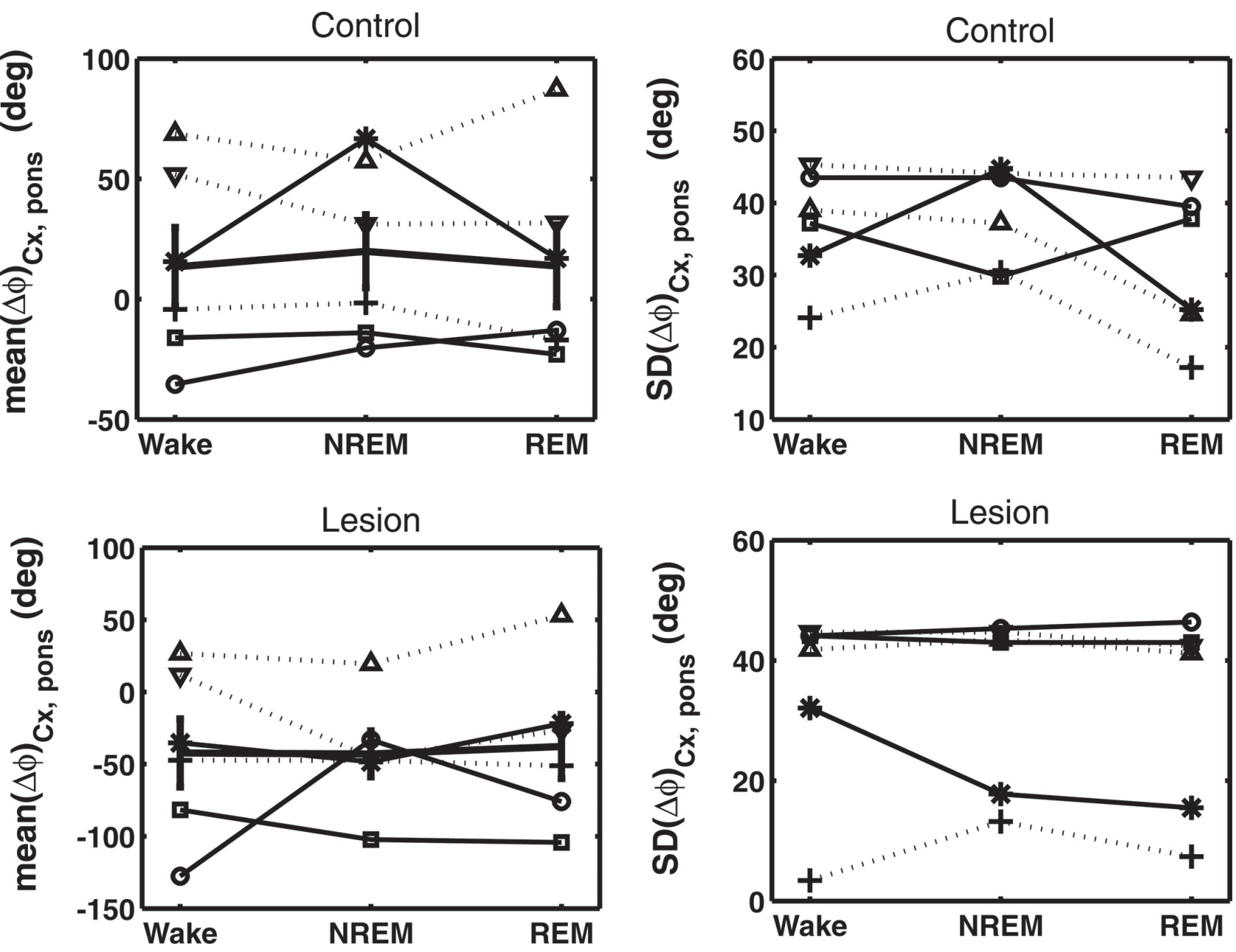

Figure 4. Sleep/wake state related mean of the cortico-pontine theta carrier frequency phase shift $\Delta \Phi$ values (left column) and their corresponding angular standard deviations (right column) for each of the six analyzed animals (individual rats are presented as different symbols), in control and lesion condition. Solid lines: DSP-4 lesion rats; dashed lines: PCA lesion rats. Bold line in the middle of the upper and lower left panels: group mean \pm SD. Negative $\Delta \Phi$ corresponds to pons leading ahead of cortex.

ences between control and lesion conditions are depicted on Table 1.

\section{Discussion}

Our results have shown that tonically decreased level of brain monoamines, caused by systemically induced chemical axotomy of the noradrenergic efferents of LC, and serotonerigic efferents of DR, in freely moving rats, increased theta synchronization phase shift between sensorimotor cortex and pons, and pons became a leading theta oscillator, generally across all sleep/wake states.

Our previous studies demonstrated that tonically decreased level of brain monoamines did not change sleep/wake states distribution during $6 \mathrm{~h}$ of sleep recording (Saponjic et al. 2007b), but it is expressed as an altered strength and mode of coupling between sigma and theta oscillations within the sensorimotor cortex, particularly with an emerged new "mode" of cortical sigma/theta oscillations coupling during NREM sleep (Kesic et al. 2011). These results suggested an important role of monoamines in NREM consolidation, and in control of the sleep spindle and theta rhythm generators. Also, loss of monoaminergic signaling altered the carrier frequency phase shift between sensorimotor cortical and pontine theta oscillations (Kalauzi et al.2009), and pons took a leading theta oscillatory role, showing that monoamines play an important role in the freely moving rat brain theta synchronization.

Hippocampal theta rhythm, in addition to other physiological functions, may be considered as a tonic REM sign, alongside with increased EEG activity and muscle atonia. 
Table 1. Lesion-induced changes of cortico-pontine theta carrier frequency phase shift $(\Delta \Phi)$ and corresponding time delays $\left(t_{D}\right)$ during Wake, NREM and REM state

\begin{tabular}{|c|c|c|c|c|c|c|c|c|}
\hline \multirow{2}{*}{ State } & \multirow{2}{*}{ Group } & \multirow{2}{*}{$\mathrm{cf}_{\theta}(\mathrm{Hz})$} & \multicolumn{2}{|c|}{ mean $\Delta \Phi(\mathrm{deg})$} & \multirow{2}{*}{$\begin{array}{c}\Delta \Phi_{\mathrm{C}}-\Delta \Phi_{\mathrm{L}} \\
\quad(\mathrm{deg})\end{array}$} & \multicolumn{2}{|c|}{$\mathrm{t}_{\mathrm{D}}(\mathrm{ms})$} & \multirow{2}{*}{$\Delta \mathrm{t} \theta(\mathrm{ms})$} \\
\hline & & & control & lesion & & control & lesion & \\
\hline \multirow{6}{*}{ Wake } & R05 (PCA) & 6.8 & $-4.3 \pm 24.1$ & $-47.3 \pm 3.4$ & 43.0 & -1.7 & -19.3 & 17.6 \\
\hline & R07 (PCA) & 6.8 & $68.7 \pm 39.0$ & $26.7 \pm 41.8$ & 42.0 & 28.1 & 10.9 & 17.2 \\
\hline & R11 (PCA) & 6.6 & $51.8 \pm 45.3$ & $11.9 \pm 44.7$ & 39.9 & 21.8 & 5.0 & 16.8 \\
\hline & R04 (DSP-4) & 5.2 & $-35.4 \pm 43.5$ & $-127.7 \pm 44.1$ & 92.3 & -18.9 & -68.2 & 49.3 \\
\hline & R06 (DSP-4) & 6.6 & $15.6 \pm 32.7$ & $-35.2 \pm 32.1$ & 50.8 & 6.6 & -14.8 & 21.4 \\
\hline & R14 (DSP-4) & 6.0 & $-16.1 \pm 37.2$ & $-81.7 \pm 44.1$ & 65.6 & -7.5 & -37.8 & 30.3 \\
\hline \multirow{6}{*}{ NREM } & R05 (PCA) & 6.8 & $-1.6 \pm 30.4$ & $-47.5 \pm 13.2$ & 45.9 & -0.65 & -19.4 & 18.7 \\
\hline & R07 (PCA) & 6.8 & $57.3 \pm 37.2$ & $19.4 \pm 43.5$ & 37.9 & 23.4 & 7.9 & 15.5 \\
\hline & R11 (PCA) & 6.6 & $31.1 \pm 44.1$ & $-45.5 \pm 44.7$ & 76.6 & 13.1 & -19.1 & 32.2 \\
\hline & R04 (DSP-4) & 5.2 & $-20.2 \pm 43.5$ & $-33.1 \pm 45.3$ & 12.9 & -10.8 & -17.7 & 6.9 \\
\hline & R06 (DSP-4) & 6.6 & $66.7 \pm 44.7$ & $-48.4 \pm 17.8$ & 115.1 & 28.1 & -20.4 & 48.5 \\
\hline & R14 (DSP-4) & 6.0 & $-14.0 \pm 29.8$ & $-102.2 \pm 43.0$ & 88.2 & -6.5 & -47.3 & 40.8 \\
\hline \multirow{6}{*}{ REM } & R05 (PCA) & 6.8 & $-16.9 \pm 17.2$ & $-51 \pm 7.4$ & 34.1 & -6.9 & -20.8 & 13.9 \\
\hline & R07 (PCA) & 6.8 & $87.1 \pm 24.6$ & $53.2 \pm 41.2$ & 33.9 & 35.6 & 21.7 & 13.9 \\
\hline & R11 (PCA) & 6.6 & $31.7 \pm 43.5$ & $-26.6 \pm 42.4$ & 58.3 & 13.3 & -11.2 & 24.5 \\
\hline & R04 (DSP-4) & 5.2 & $-12.9 \pm 39.5$ & $-75.9 \pm 46.4$ & 63.0 & -6.9 & -40.5 & 33.6 \\
\hline & R06 (DSP-4) & 6.6 & $16.9 \pm 25.2$ & $-21.9 \pm 15.5$ & 38.8 & 7.1 & -9.2 & 16.3 \\
\hline & R14 (DSP-4) & 6.0 & $-22.9 \pm 37.8$ & $-104.3 \pm 43.0$ & 81.4 & -10.6 & -48.3 & 37.7 \\
\hline
\end{tabular}

On the other hand, PGO (ponto-geniculo-occipital) waves represent the most important REM phasic sign, although a number of other phenomena may be simultaneously detected (muscle twitches, breathing and heart rate fluctuations, rapid eye movements). Before and during REM phase, a group of neurons in the pontine tegmentum is subject to a phasic activation, causing the appearance of the electro-

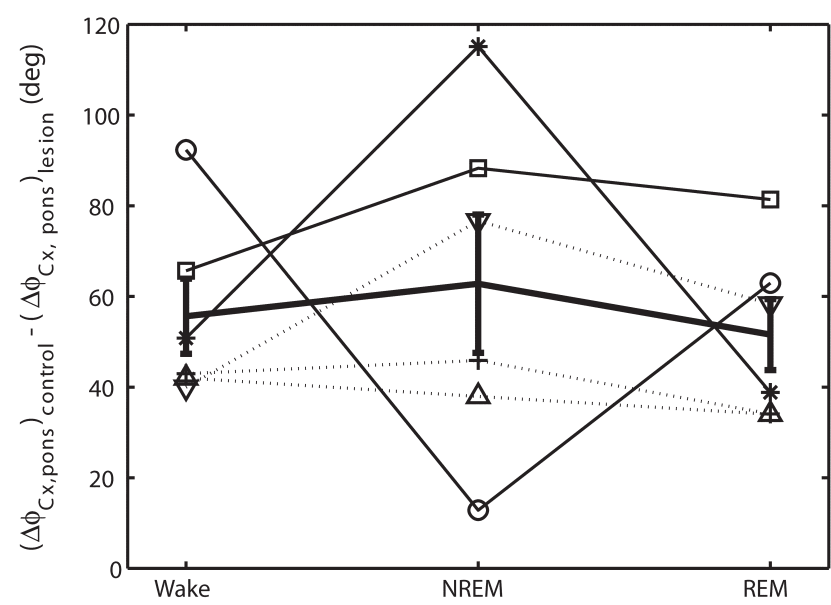

Figure 5. Sleep/wake states related differences of the mean $\Delta \Phi$ values, between control and lesion conditions, for the same group of rats depicted in two left panels at Fig. 4. physiologically detectable PGO waves (or corresponding P wave at the pontine level). Glutamatergic neurons of locus subcoeruleus are thought to be the $\mathrm{P}$ wave generator (Datta et al. 1998; Datta and Mac Lean 2007). Projections of this nucleus include PPT, hippocampus, amygdala, enthorinal and visual cortex (Datta et al. 1998). Within these, PPT is significant as a part of the ascending brainstem hippocampal synchronizing pathway, while its projection to dorsal hippocampus has been pharmacologically proven (Datta 2006). During REM phase, a positive correlation was found between $\mathrm{P}$ wave activity and hippocampal theta waves (Karashima et al. 2004, 2005, 2007). In order to explain detected phase locking between hippocampal theta rhythm and the $\mathrm{P}$ waves during REM sleep in rats, Karashima et al. (2004) offered three hypotheses: $P$ wave and REM generators control theta frequency and amplitude; theta wave generator controls $\mathrm{P}$ wave and REMs densities; both theta wave dynamics and $\mathrm{P}$ wave and REMs densities are controlled by some other common mechanisms.

Since all REM phasic phenomena, such as the muscle twitches, respiration and heart rhythm surges and $\mathrm{P}$ waves, tended to occur simultaneously, Karashima et al. (2004) hypothesized that they are all linked to one common phasic event generator in the brain. On the bases of his study which was in agreement with other studies (Datta and Patterson 2003; Saponjic et al. 2003), PPT was suggested as a possible pontine reticular formation structure influencing the 
dynamics of the phasic events, as well as the hippocampal theta waves.

In our former study we have found (Kalauzi et al. 2009) the significant cortico-pontine theta carrier frequency phase shift locking, and a systemic monoaminegic lesion induced pontine leading role in the brain theta oscillations. Our present study evidenced that the angular phase shift increase fell in the range of 27.1-86.9 degrees $(57.37 \pm 24.44 \mathrm{deg})$, corresponding to temporal delay in the range of $14.5-40.2 \mathrm{~ms}(25.30 \pm 10.54 \mathrm{~ms})$ across the population.

We have shown that this monoaminergic lesion induced theta carrier frequency phase shift $(\Delta \Phi)$ increase between pons and cortex is generalized, and non specific for any sleep/wake state (Wake, NREM and REM; Fig. 5). Still, although not significant, the monoaminergic lesions (lesion of the brain LC and DR afferent systems) induced the highest group mean $\Delta \Phi$ difference during NREM sleep, probably due to lesion induced more frequent NREM/REM transitions. Although in this study we did not differentiate the transition states during sleep, the NREM/REM transitions are states with predominance of the phasic theta versus tonic theta oscillations, and are characterized with an increase of $\mathrm{P}$ wave densities, particularly clustered $\mathrm{P}$ waves, whose intrinsic frequency corresponds to theta frequency oscillations (Datta and McLean 2007), and could additionally yield higher NREM $\Delta \Phi$ difference.

Acknowledgements. This work was supported by project OI173022, financed by the Serbian Ministry of Education and Science, and by NIH Grant AG016303.

\section{References}

Bland B. H., Oddie S. D. (1998): Anatomical, electrophysiological and pharmacological studies of ascending brainstem hippocampal synchronizing pathways. Neurosci. Biobehav. Rev. 22, $259-273$ http://dx.doi.org/10.1016/S0149-7634(97)00013-4

Buzsaki G. (2002): Theta oscillations in the hippocampus. Neuron 31, 325-340 http://dx.doi.org/10.1016/S0896-6273(02)00586-X

Carley W. D., Radulovacki M. (2003): Pathophysiology of sleeprelated breathing disorders: unanswered questions. In: Sleeprelated Breathing Disorders: Experimental Models and Therapeutic Potential. (Eds. W. D. Carley and M. Radulovacki), pp. 3-11, Marcel Dekker Inc., New York

Datta S., Siwek D. F., Patterson E. H., Cipolloni P. B. (1998): Localization of pontine PGO wave generation sites and their anatomical projections in the rat. Synapse 30, 409-423 http://dx.doi.org/10.1002/(SICI)1098-2396(199812)30:4<409:: AID-SYN8>3.0.CO;2-\#

Datta S., Patterson E. H. (2003): Activation of phasic pontine wave (P-wave): a mechanism of learning and memory processing. In: Sleep and Brain Plasticity. (Eds. P. Maquet,
C. Smith and R. Stickgold), pp. 135-156, Oxford University Press, New York

http://dx.doi.org/10.1093/acprof:oso/9780198574002.003.0008

Datta S. (2006): Activation of phasic pontine-wave generator: a mechanism for sleep-dependent memory processing. Sleep. Biol. Rhythms 4, 16-26

http://dx.doi.org/10.1111/j.1479-8425.2006.00202.x

Datta S., MacLean R. R. (2007): Neurobiological mechanisms for the regulation of mammalian sleep-wake behavior: reinterpretation of historical evidence and inclusion of contemporary cellular and molecular evidence. Neurosci. Biobehav. Rev. 31, $775-824$

http://dx.doi.org/10.1016/j.neubiorev.2007.02.004

Fenik P., Veasey S. C. (2003): Pharmacological characterization of serotonergic receptor activity in the hypoglossal nucleus. Am. J. Respir. Crit. Care Med. 167, 563-569 http://dx.doi.org/10.1164/rccm.200202-107OC

Hobson J. A., McCarley R. W., Wyzinski P. W. (1975): Sleep cycle oscillation: reciprocal discharge by two brainstem neuronal groups. Science 189, 55-58 http://dx.doi.org/10.1126/science.1094539

Kalauzi A., Kesic S., Rakic Lj., Saponjic J. (2008): Changes in cortical and pontine sigma and theta amplitude oscillations coupling following monoaminergic lesions in rat. Revista Espanola De Neuropsicologia 10, 134-135

Kalauzi A., Kesic S., Saponjic J. (2009): Cortico-pontine theta synchronization phase shift following monoaminergic lesion in rat. J. Physiol. Pharmacol. 60, 79-84

Karashima A., Nakao M., Honda K., Iwasaki N., Katayama N., Yamamoto M. (2004): Theta wave amplitude and frequency are differentially correlated with pontine waves and rapid eye movements during REM sleep in rats. Neurosci. Res. 50, 283-289 http://dx.doi.org/10.1016/j.neures.2004.07.007

Karashima A., Nakao M., Katayama N., Honda K. (2005): Instantaneous acceleration and amplification of hippocampal theta wave coincident with phasic activities during REM sleep. Brain Res. 1051, 50-56 http://dx.doi.org/10.1016/j.brainres.2005.05.055

Karashima A., Katayama N., Nakao M. (2007): Phase-locking of spontaneous and tone-elicited pontine waves to hippocampal theta waves during REM sleep in rats. Brain Res. 1182, 73-81 http://dx.doi.org/10.1016/j.brainres.2007.08.060

Kesic S., Kalauzi A., Radulovacki M., Carley W. D., Saponjic J. (2011): Coupling changes in cortical and pontine sigma and theta frequency oscillations following monoaminergic lesions in rat. Sleep Breath. 15, 35-47 http://dx.doi.org/10.1007/s11325-010-0327-6

Lydic R., McCarley R. W., Hobson J. A. (1987): Serotonin neurons and sleep. Time course of dorsal raphe discharge, PGO waves, and behavioral states. Arch. Ital. Biol. 126, 1-28

McCarley R. W., Hobson J. A. (1975): Neuronal excitability modulation over the sleep cycle: a structural and mathematical model. Science 189, 58-60 http://dx.doi.org/10.1126/science.1135627

Penttonen M., Buzsaki G. (2003): Natural logarithmic relationship between brain oscillators. Thalamus Relat. Syst. 2, 145-152 
Saponjic J., Radulovacki M., Carley D. W. (2003): P-waves are coupled to long-lasting respiratory disturbance following glutamate stimulation of the pedunculo-pontine tegmental nucleus (PPT) Sleep, Suppl. 26, A45-46

Saponjic J., Cvorovic J., Radulovacki M., Carley D. W. (2005) Serotonin and noradrenaline modulate respiratory pattern disturbances evoked by glutamate injection into the pedunculopontine tegmentum of anesthetized rats. Sleep 28, 560-570

Saponjic J., Kalauzi A., Radulovacki M., Carley D. W. (2007a): Ponto-cortico-muscular oscillation path following the lesion of serotonergic axons in rat. Neuroscience today: neuronal functional diversity and collective behaviors, Florence 26-28 March, 27-31

Saponjic J., Radulovacki M., Carley D. W. (2007b): Monoaminergic system lesions increase post-sigh respiratory pattern disturbance during sleep in rats. Physiol. Behav. 90, 1-10 http://dx.doi.org/10.1016/j.physbeh.2006.08.019
Singer W. (1993): Synchronization of cortical activity and its putative role in information processing and learning. Annu. Rev. Physiol. 55, 349-374

http://dx.doi.org/10.1146/annurev.ph.55.030193.002025

Vertes R. P. (1982): Brainstem generation of hippocampal EEG. Prog. Neurobiol. 19, 159-186

http://dx.doi.org/10.1016/0301-0082(82)90005-3

Vertes R. P., Kocsis B. (1997): Brainstem-diencephalo-septohippocampal systems controlling the theta rhythm of the hippocampus. Neuroscience 81, 893-926

Vinogradova O. S. (1995): Expression, control, and probable functional significance of the neuronal theta-rhythm. Prog. Neurobiol. 45, 523-583

http://dx.doi.org/10.1016/0301-0082(94)00051-I

Received: September 13, 2011

Final version accepted: January 18, 2012 